\title{
Tachyon constant-roll inflation
}

\author{
A. Mohammadi, ${ }^{*}$ Kh. Saaidi, ${ }^{\dagger}$ and T. Golanbari ${ }^{\ddagger}$ \\ Department of Physics, Faculty of Science, University of Kurdistan, \\ Pasdaran Street, P.O. Box 66177-15175, Sanandaj, Iran
}

(Received 12 February 2018; published 17 April 2018)

\begin{abstract}
The constant-roll inflation is studied where the inflaton is taken as a tachyon field. Based on this approach, the second slow-roll parameter is taken as a constant which leads to a differential equation for the Hubble parameter. Finding an exact solution for the Hubble parameter is difficult and leads us to a numerical solution for the Hubble parameter. On the other hand, since in this formalism the slow-roll parameter $\eta$ is constant and could not be assumed to be necessarily small, the perturbation parameters should be reconsidered again which, in turn, results in new terms appearing in the amplitude of scalar perturbations and the scalar spectral index. Utilizing the numerical solution for the Hubble parameter, we estimate the perturbation parameter at the horizon exit time and compare it with observational data. The results show that, for specific values of the constant parameter $\eta$, we could have an almost scale-invariant amplitude of scalar perturbations. Finally, the attractor behavior for the solution of the model is presented, and we determine that the feature could be properly satisfied.
\end{abstract}

DOI: 10.1103/PhysRevD.97.083006

\section{INTRODUCTION}

Guth's proposal in 1981 for solving the problem of the big bang theory is now one of the best candidates for describing the Universe's evolution in its earliest time [1]. Although his scenario, known as old inflation, did not work properly, his idea has been followed by physicists and many different scenarios of inflation have been introduced, such as new inflation [2,3], chaotic inflation [4], k-inflation $[5,6]$, brane inflation [7,8], G-inflation [9-12] warm inflation [13-19], and so on.

Inflation is known as an accelerated expansion phase in the very early time when the Universe experienced an extreme expansion in a very short period of time. Commonly, the inflation is derived by a scalar field, which varies very slowly to give a quasi-de Sitter expansion. Such models of the inflationary scenario are known as slow-roll inflation and are described by slow-roll parameters. In order to allow enough expansion, these parameters should be much smaller than unity especially in the early stage of the Universe [20]. The most well-known slow-roll parameters are $\epsilon=-\dot{H} / H^{2}$, the smallness of which means that

\footnotetext{
*abolhassanm@gmail.com, a.mohammadi@uok.ac.ir †ksaaidi@uok.ac.ir

t.golanbari@gmail.com
}

Published by the American Physical Society under the terms of the Creative Commons Attribution 4.0 International license. Further distribution of this work must maintain attribution to the author(s) and the published article's title, journal citation, and DOI. Funded by SCOAP ${ }^{3}$. the fractional change of the Hubble parameter during Hubble time is much smaller than unity. Usually, the same assumption is taken for the time derivative of the scalar field; namely, the fractional change of the $\dot{\phi}$ during Hubble time (denoting by $\eta=\ddot{\phi} / H \dot{\phi}$ ) should be much smaller than unity [21].

As long as the condition $\epsilon<1$ is true, the Universe remains in an accelerated phase, and the smallness of $\eta$ ensures we will have enough inflation in order to overcome the problems of the hot big bang model. It is stated that the smallness of these parameters is provided by an almost flat part of the scalar field potential, which in turn results in an almost scale-invariant spectrum of scalar perturbations. However, what happens if the potential is exactly flat? This question was explored in [22] for the first time. In [23], the non-Gaussianity of this situation was explored in detail, and they showed that in contrast to the standard slow-roll inflation, the non-Gaussianity could be of order unity. The idea was generalized in [24] so that the second slow-roll parameter was taken as a constant that could be of order unity. They showed that the power spectrum on the superhorizon could be both growing and scale invariant and that the non-Gaussianity of the model could be of order unity too. The model was addressed as ultra-slow-roll inflation. The same model was studied in [25], where the author found an exact solution for the Hubble parameters and potential using Hamilton-Jacobi formalism [26-32]. It was determined that the model has an attractor solution, and that the power spectrum remains scale invariant for specific choices of constant parameter. The standard model for describing inflation could be 
generalized in different ways; refer to [33] for more detail. Reference [34] could be called the first generalized case of the scenario, where the application of constant-roll inflation in modified gravity has been investigated using two approaches. The first approach involves a conformal transformation to go to Einstein frame scalar-tensor theory. The consideration shows that the corresponding scalar potential is different. The second approach does not involve scalartensor theory. The authors derived the slow-roll parameters for a general form of $f(R)$, then they considered in detail two specific choices of $f(R)$ : Starobinsky $R^{2}$ and powerlaw $f(R)$. The model predicted results that the scalar spectral index and the tensor-to-scalar ratio are acceptable in comparison with observational data. Motahashi and Starobinsky considered constant-roll inflation in $f(R)$ gravity [35], which leads to a scalar kinetic term by a conformal transformation. They relate the time coordinate and scale factor in the Jordan and Einstein frame, and the Einstein equation and Klein-Gordon equation were derived in the Einstein frame. By generalizing the constant-roll condition to $\ddot{F}=\beta H \dot{F}$, the modified differential equation was obtained for the Hubble parameter. The solution for the Hubble parameter shows that, unlike [25], the Hubble parameter and, in turn, the potential are not periodic. After calculating the perturbation parameters, they found that only for $\beta<0$ could the observational constraint be satisfied. The constant-roll inflationary scenario in $f(T)$ teleparallel gravity has been investigated in [36]. The authors could nicely define the slow-roll parameters, where by taking the second slow-roll parameter as a constant, i.e. $\ddot{\phi} / H \dot{\phi}=\beta$, they recover the ultra-slow-roll case for $\beta=-3$. Because of modifying the gravity theory, the differential equation of the Hubble parameter and also the potential of the scalar field are modified as well. By taking the Hubble parameter as in [motahashi], they reconstruct the $f(T)$ gravity and study the behavior of the potential for different choices of the constant $\beta$ and also investigate the related cosmic evolution. After that, a power-law function of the torsion scalar is introduced for $f(T)$, and by considering the perturbation parameters of the model, it was clarified that the model could be in good agreement with observational data. Some other interesting work about the subject can be found in [37-41]. A more generalized case has been considered in [42], where instead of assuming a constant for $\eta$, it was taken as equal to a smooth function of the scalar field. Such a model is addressed as smooth-roll inflation in [42-44].

In this paper, the scenario of constant-roll inflation will be considered for the tachyon field. Applications of the tachyon field in cosmology received much attention after the work of Sen [45-47] and Gibbons [48]. Sen showed that pressureless gas will be produced during the process of the classical decay of unstable D-branes in string theory. The starting point of tachyon cosmology was the work of Gibbons [48], where the Einstein-Hillbert term was added to the effective action of the tachyon on the brane as a way to take into account the coupling to the gravitational field. Since it is assumed that inflation may be caused by a scalar field (inflaton) with negative pressure and because of the fact that there is no reason not to take the inflaton as tachyon field, tachyon inflation becomes an interesting topic amongst physicists [49-52]. This huge interest in tachyon slow-roll inflation motivate us to consider the scenario of tachyon inflation for a more generalized case.

The paper is organized as follows: The general formula of the model is addressed in Sec. II. Applying tachyon field as a inflaton for describing inflation is presented in Sec. III, where the dynamical equations are obtained based on constant-roll condition. In Sec. IV, the scalar and tensor perturbations of the model is extracted and the power spectrum of perturbations are achieved in compatible with constant-roll condition. Agreement of the model prediction with observational data is examined in Sec. V. In Sec. VI, another choice is assumed for the second slow-roll parameter. The attractor behavior of the solution will be considered in Sec. VII for both cases. The result of the work is summarized in Sec. VI.

\section{TACHYON MODEL}

The action for the model is given by

$S=\int d^{4} x \sqrt{-g}\left(\frac{-1}{16 \pi G} R-V(T) \sqrt{1-g^{\mu \nu} \nabla_{\mu} T \nabla_{\nu} T}\right)$,

where $T$ stands for tachyon field with potential $V(T)$. The field equation of the model that is derived by variation with respect to the metric is read as

$$
\begin{aligned}
R_{\mu \nu}-\frac{1}{2} g_{\mu \nu} R= & 8 \pi G\left(-V(T) g_{\mu \nu} \sqrt{1-\nabla_{\alpha} T \nabla^{\alpha} T}\right. \\
& \left.+\frac{V(T)}{\sqrt{1-\nabla_{\alpha} T \nabla^{\alpha} T}} \nabla_{\mu} T \nabla_{\nu} T\right),
\end{aligned}
$$

in which the term in parentheses on the right-hand side is known as the energy-momentum tensor of tachyon field. Taking the tachyon field as a perfect fluid with energy-momentum tensor $T_{\mu \nu}=(\rho+p) u_{\mu} u_{\nu}-p g_{\mu \nu}$, one could realize that the four-velocity of the tachyon is $u_{\mu}=\nabla_{\mu} T /\left(-\nabla_{\alpha} T \nabla^{\alpha} T\right)$. The equation of motion of tachyon field is derived by tacking variation of action (1) with respect to field $T$, as

$$
\nabla_{\mu} \nabla^{\mu} T+\frac{\nabla^{\mu} T \nabla^{\nu} T}{\left(1-\nabla_{\alpha} T \nabla^{\alpha} T\right)} \nabla_{\mu} \nabla_{\nu} T+\frac{V_{, T}(T)}{V(T)}=0 .
$$

In a homogenous and isotropic universe described by a flat FLRW metric, the main evolution equations of the models are obtained as 


$$
H^{2}=\frac{8 \pi G}{3} \rho_{T}, \quad \dot{H}=-4 \pi G\left(\rho_{T}+p_{T}\right),
$$

where $\rho_{T}$ and $p_{T}$ are, respectively, the energy density and pressure of tachyon field given by

$$
\rho_{T}=\frac{V(T)}{\sqrt{1-\dot{T}^{2}}}, \quad p_{T}=-V(T) \sqrt{1-\dot{T}^{2}} .
$$

From Eq. (4), the acceleration equation is obtained as $\ddot{a} / a=$ $H^{2}\left(1-3 \dot{T}^{2} / 2\right)$, then the Universe stays in an acceleration expansion phase as long as the condition $\dot{T}^{2}<2 / 3$; otherwise, the Universe decelerates. Note that for the case $\dot{T}=0$, we have exactly cosmological constant equation of motion parameter for the scalar field i.e. $\omega=\rho_{T} / p_{T}=-1$.

The second order equation for tachyon field comes from (3)

$$
\ddot{T}+\left(1-\dot{T}^{2}\right)\left(3 H \dot{T}+\frac{V_{, T}(T)}{V(T)}\right)=0,
$$

which is another statement of conservation equation $\dot{\rho}_{T}+3 H\left(\rho_{T}+p_{T}\right)=0$ that also could be concluded form Friedmann equations (4). If we use the Hamilton-Jacobi formalism and assume the Hubble parameter as a function of tachyon field, namely $H:=H(T)$, from the Friedmann equation (4), one obtains

$$
\dot{T}=-\frac{2}{3} \frac{H_{, T}(T)}{H^{2}(T)} .
$$

Substituting Eq. (7) in Friedmann equation (4), and using Eq. (5), the potential of tachyon field is derived as

$$
V(T)=\frac{3}{8 \pi G} H^{2}(T) \sqrt{1-\frac{4}{9} \frac{H_{T}^{2}(T)}{H^{4}(T)}} .
$$

From now on we take $M_{p}^{2}=1 / 8 \pi G=1$.

\section{TACHYON INFLATION}

The smallness of the first slow-roll parameter should be guaranteed to have an accelerated phase. Applying the Hamilton-Jacobi formalism, and taking the Hubble parameter as a function of scalar field, one arrives at

$$
\epsilon=-\frac{\dot{H}}{H^{2}}=-\frac{\dot{T} H_{, T}(T)}{H^{2}(T)}=\frac{2}{3} \frac{H_{, T}^{2}(T)}{H^{4}(T)} .
$$

In constant-roll inflation, the rate of time derivative of field is taken as a constant, namely

$$
\ddot{T}=\beta H \dot{T},
$$

then, our second slow-roll parameter $\eta$ is obtained by
$\eta=\frac{\ddot{T}}{H \dot{T}}=-\delta+2 \epsilon=\beta, \quad$ where $\delta=\frac{2}{3} \frac{H_{, T T}(T)}{H^{3}(T)}$.

Using Eqs. (7) and (10), the differential equation for the Hubble parameter is given by

$$
H(T) \frac{d^{2} H(T)}{d T^{2}}-2\left(\frac{d H(T)}{d T}\right)^{2}+\frac{3}{2} \beta H^{4}(T)=0,
$$

which is a nonlinear second order differential equation; and it is more complicated than the corresponding equation that in [25] was derived. Getting analytical solution for the above differential equation is at least very complicated, then we solve it using the numerical approach for specific choice of the parameter $\beta$, illustrated in Fig. 1 where the Hubble parameter decreases by enhancement of the tachyon field.

Applying this solution, the behavior of slow-roll parameters $\epsilon$ and $\delta$ will be determined during the inflationary times. Figure 2(a) illustrates the behavior of $\epsilon$ versus the tachyon field. The parameter is much smaller than unity at the beginning of inflation and grows up by passing time. It reaches one for larger values of the tachyon field. The slow-roll parameter $\delta$ is depicted in Fig. 2(b), where one can see that the parameter is close to $\beta$ (not necessarily small) at the beginning of inflation, the result that we expected from Eq. (11).

From Eq. (8), the potential of tachyon field is extracted versus the field. Figure 3 displays the tachyon potential so that The field rolls down from the top of the potential.

The amount of inflationary expansion during this short period of time is important in inflationary studies. The parameter for describing this feature is known as the number of $e$-folds and is given by

$$
N=\int_{T}^{T_{e}} H d t=-\frac{3}{2} \int_{T}^{T_{e}} \frac{H^{3}(T)}{H_{, T}(T)} d t
$$

where $T_{e}$ indicates the value of the field at the end of inflation. To solve the horizon and flatness problem, we need about $60 e$-folds [33].

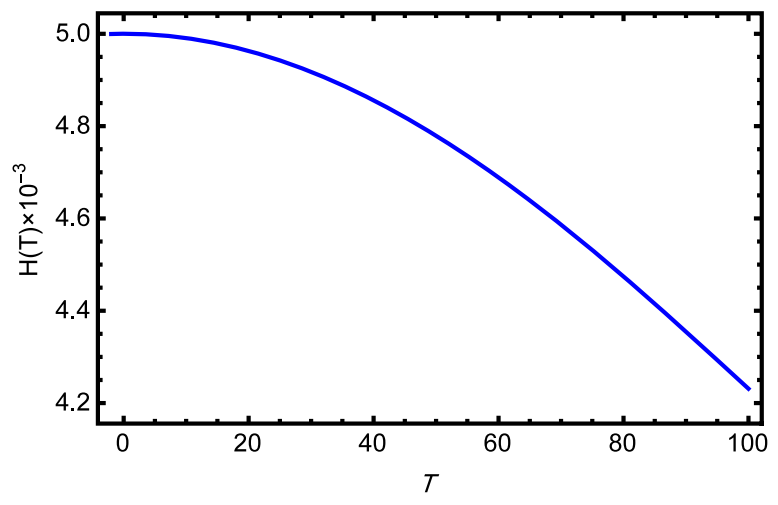

FIG. 1. The Hubble parameter versus the tachyon field. 

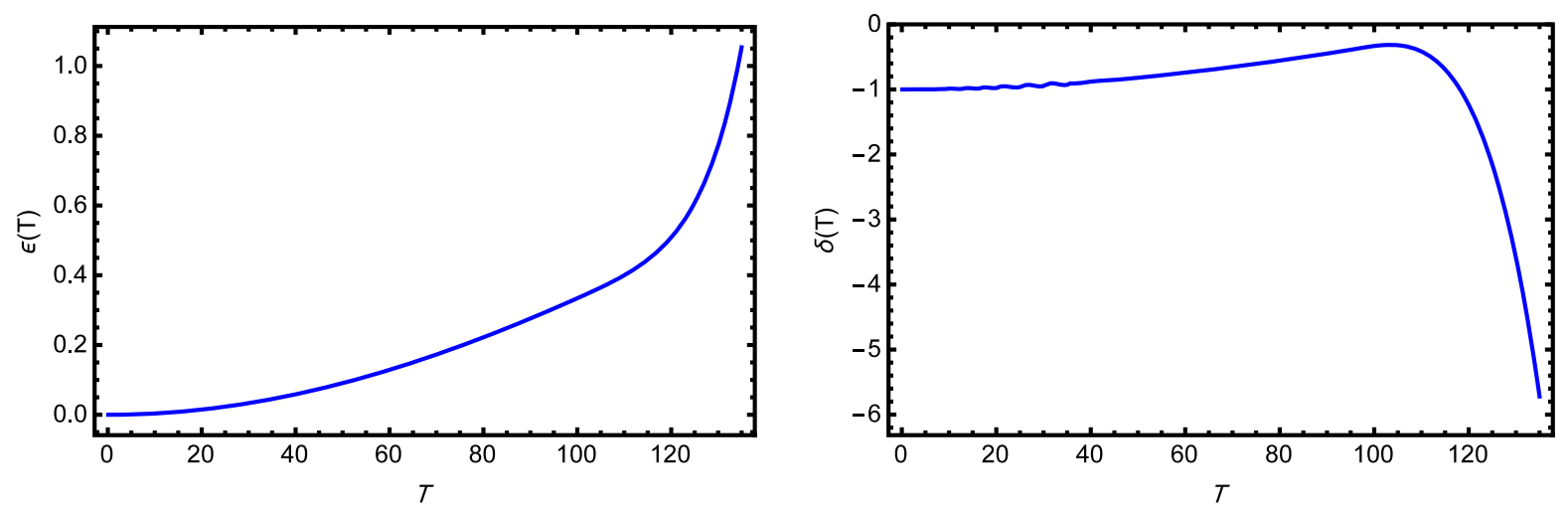

FIG. 2. The behavior of the slow-roll parameters $\epsilon$ and $\delta$ during the inflationary times versus tachyon field.

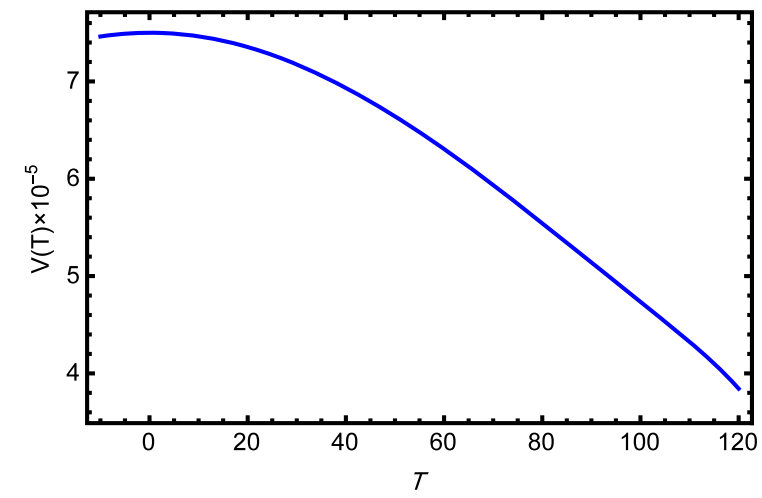

FIG. 3. The tachyon field potential versus the field in the inflationary times.

\section{PERTURBATIONS}

Quantum perturbations are another prediction of the inflationary scenario. During the inflationary phase, the fluctuations are stretched and go beyond the horizon. At superhorizon scale, the fluctuations are frozen and reenter the horizon after inflation. The footprint of the fluctuations are in the cosmic microwave background (CMB), which is emitted freely in the Universe at the last surface scattering. Therefore, CMB is our best chance to understand the evolution in the early time of the Universe. Temperature fluctuations in CMB are related to the metric fluctuations, which in turn are a consequence of scalar field fluctuation (here, tachyon field fluctuations). Metric fluctuations, in general, are divided into three types: scalar, vector, and tensor fluctuations which at linear order evolve separately. The scalar fluctuations are known as seeds for the large-scale structure of the Universe, and tensor fluctuations are addressed as gravitational waves of the early Universe. Vector fluctuations are diluted during inflation and are not our interested perturbations mode. Then, the most important fluctuation types are scalar and tensor fluctuation. In this section, we first consider the scalar fluctuations, and after that we introduce the tensor fluctuations.

\section{A. Scalar perturbation}

Consider a small fluctuation of the tachyon field as

$$
T(t, \mathbf{x})=T_{0}(t)+\delta T(t, \mathbf{x}),
$$

where the index " 0 " indicates that the parameter is a background parameter. The fluctuations in the tachyon field cause fluctuations in the metric so that the scalar type of these fluctuations in longitudinal gauge is

$$
d s^{2}=(1+2 \Phi(t, \mathbf{x})) d t^{2}-a^{2}(t)(1-2 \Phi(t, \mathbf{x})) \delta_{i j} d x^{i} d x^{j} .
$$

Following [6,53], the linearized Einstein equation could be written as

$$
\begin{aligned}
& \dot{\xi}=\frac{a\left(\rho_{T}+p_{t}\right)}{H^{2}} \zeta, \\
& \dot{\zeta}=\frac{c_{s}^{2} H^{2}}{a^{3}\left(\rho_{T}+p_{t}\right)} \nabla^{2} \xi
\end{aligned}
$$

in which the new variables $\xi$ and $\zeta$ are defined as [6]

$$
\begin{aligned}
\xi & \equiv \frac{a}{4 \pi G H} \Phi, \\
\zeta & \equiv \frac{4 \pi G H}{a} \xi+H \frac{\delta T}{\dot{T}}=\Phi+H \frac{\delta T}{\dot{T}} .
\end{aligned}
$$

The corresponding action for Eq. (14) is [6]

$$
S=\frac{1}{2} \int z^{2}\left(\zeta^{\prime 2}+c_{s}^{2} \zeta(\nabla \zeta)^{2}\right) d \tau d^{3} \mathbf{x}
$$

and the parameter $z$ is given by $z=\sqrt{3 / 8 \pi G}\left(a \dot{T} / c_{s}\right)$. The prime in the above equation means derivative with respect to the conformal time $\tau$. Introducing a canonical quantization variable $v=z \zeta$, the action (15) is rewritten in terms of $v$, which is known as the Mukhanov-Sasaki variable: 


$$
S=\frac{1}{2} \int\left(v^{2}+c_{s}^{2} v(\nabla v)^{2}+\frac{z^{\prime \prime}}{z} v\right) d \tau d^{3} \mathbf{x} .
$$

Therefore, we have

$$
\frac{d^{2}}{d \tau^{2}} v(\tau, \mathbf{x})-c_{s}^{2} \nabla^{2} v(\tau, \mathbf{x})-\frac{z^{\prime \prime}}{z} v(\tau, \mathbf{x})=0
$$

The conformal time derivatives of $z$ is extracted as

$$
\frac{d z}{d \tau}=z(a H)\left[1+2 \epsilon-\delta+\frac{2 \epsilon}{(3-2 \epsilon)}(2 \epsilon-\delta)\right] .
$$

In order to get the second time derivative, one needs to calculate the time derivative of the slow-roll parameters,

$$
\begin{aligned}
& \frac{d \epsilon}{d \tau}=a H\left(2 \epsilon \delta-4 \epsilon^{2}\right), \\
& \frac{d \delta}{d \tau}=a H\left(\sigma^{2}-3 \epsilon \delta\right),
\end{aligned}
$$

where $\sigma=2 \sqrt{H^{\prime} H^{\prime \prime \prime}} / 3 H^{3}$. Then, the second order derivative of $z$ with respect to the conformal time becomes

$$
\begin{aligned}
\frac{1}{z} \frac{d^{2} z}{d \tau^{2}}= & 2(a H)^{2}\left[\left(1+\frac{5}{2} \epsilon-\frac{3}{2} \delta-3 \epsilon^{2}+\frac{1}{2} \delta^{2}+2 \epsilon \delta-\frac{1}{2} \sigma^{2}\right)\right. \\
& +\frac{\epsilon}{(3-2 \epsilon)}\left(4 \epsilon-2 \delta-8 \epsilon^{2}+7 \epsilon \delta-\sigma^{2}\right) \\
& \left.+\frac{2 \epsilon^{2}}{3(3-2 \epsilon)^{2}}\left(-4 \epsilon^{2}+4 \epsilon \delta-\delta^{2}\right)\right] .
\end{aligned}
$$

Now, we go back to the main equation (17). By expanding the canonical quantization variable $v$ in the Fourier modes as

$$
v(\tau, \mathbf{x})=\int \frac{d^{3} \mathbf{k}}{(2 \pi)^{3 / 2}} v_{k}(\tau) e^{i \mathbf{k} \cdot \mathbf{x}}
$$

and inserting this in Eq. (17), we arrive at [53]

$$
\frac{d^{2}}{d \tau^{2}} v_{k}(\tau)+\left(c_{s}^{2} k^{2}-\frac{1}{z} \frac{d^{2} z}{d \tau^{2}}\right) v_{k}(\tau)=0 .
$$

We need to find $v_{k}(\tau)$ from the above equation to extract the power spectrum. The power spectrum of curvature perturbations as a function of wave number $k$ is given by

$$
\mathcal{P}_{s}=\frac{k^{3}}{2 \pi^{2}}\left|\frac{v_{k}}{z}\right|^{2} .
$$

At the subhorizon scale, when $c_{s} k \gg a H$, the differential equation (18) gets a simple form from which the solution could be easily derived $[6,53]$ : $\frac{d^{2}}{d \tau^{2}} v_{k}(\tau)+c_{s}^{2} k^{2} v_{k}(\tau)=0, \Rightarrow v_{k}(\tau)=\frac{1}{\sqrt{2 c_{s} k}} e^{i c_{s} k \tau}$.

To find a general solution, we first work a little with term $z^{\prime \prime} / z$. Since the slow-roll parameters appearing in $z^{\prime \prime} / z$ are smaller than unity, except $\eta$ (or $\delta$ ), we rewrite this term up to first order of $\epsilon$ and $\sigma$, namely,

$$
\frac{1}{z} \frac{d^{2} z}{d \tau^{2}}=2(a H)^{2}\left(1+\frac{5}{2} \epsilon-\frac{3}{2} \delta+\frac{1}{2} \delta^{2}+\frac{4}{3} \epsilon \delta\right) .
$$

On the other hand, for a quasi-de Sitter expansion (which is at least acceptable for the beginning of inflation), the conformal time is derived in terms of the scale factor and Hubble parameter as $\tau=-(1+\epsilon) / a H$; therefore, there is $a^{2} H^{2} \simeq(1+\epsilon)^{2} / \tau^{2}$. Substituting this in term $z^{\prime \prime} / z$, we could have

$$
\frac{1}{z} \frac{d^{2} z}{d \tau^{2}}=\frac{\nu^{2}-\frac{1}{4}}{\tau^{2}}, \quad \nu^{2} \equiv \frac{9}{4}+9 \epsilon-3 \delta+\delta^{2}-\frac{10}{3} \epsilon \delta+2 \delta^{2} \epsilon .
$$

Inserting this in Eq. (18), and using a variable change as $v_{k}=\sqrt{-\tau} f_{k}$, the canonical form of Bessel's equation is extracted so that

$$
\frac{d^{2} f_{k}}{d x^{2}}+\frac{1}{x} \frac{d f_{k}}{d x}+\left(1-\frac{\nu^{2}}{x^{2}}\right) f_{k}=0
$$

The most general solution for the equation is the Hankel functions. Therefore, we have

$$
v_{k}(\tau)=\sqrt{-\tau}\left[\alpha_{k} H_{\nu}^{(1)}(x)+\gamma_{k} H_{\nu}^{(2)}(x)\right],
$$

where $\alpha_{k}$ and $\gamma_{k}$ are constant that generally depend on wave number $k$. The solution should approach to solution (20) on subhorizon scale (as $-k \tau \rightarrow \infty$ ). The asymptotic behavior of the Hankel functions at this limit is given by

$$
\lim _{-k \tau \rightarrow \infty} H_{\nu}^{(1,2)}(x)=\sqrt{\frac{2}{\pi}} \frac{1}{\sqrt{2 c_{s} k}} e^{\mp\left(i c_{s} k \tau+\varsigma\right)}, \quad \varsigma=\frac{1}{2}\left(\nu+\frac{1}{2}\right) .
$$

In comparison with solution (20), it is clear that $\alpha_{k}=$ $\sqrt{\pi} e^{i \delta} / 2$ and $\gamma_{k}=0$. Inserting this into Eq. (22), the general solution for variable $v_{k}(\tau)$ becomes

$$
v_{k}(\tau)=\frac{\sqrt{\pi}}{2} e^{i \frac{\pi}{2}(\nu+1 / 2)} \sqrt{-\tau} H_{\nu}^{(1)}\left(-c_{s} k \tau\right) .
$$

The scalar power-spectrum follows from Eq. (19) and is read as

$$
\mathcal{P}_{s}=\frac{1}{2 \pi} \frac{8 \pi G}{3} \frac{3 c_{s}^{2} H^{2}}{2 \epsilon}\left(\frac{k}{a H}\right)^{3}\left|H_{\nu}^{(1)}\left(-c_{s} k \tau\right)\right|^{2} .
$$


Following the asymptotic behavior of the Hankel function, the scalar power spectrum on the superhorizon scale becomes

$\mathcal{P}_{s}=\frac{8 \pi G}{3}\left(\frac{H}{2 \pi}\right)^{2} \frac{3}{2 c_{s} \epsilon}\left(\frac{2^{\nu-3 / 2} \Gamma(\nu)}{\Gamma(3 / 2)}\right)^{2}\left(\frac{c_{s} k}{a H}\right)^{3-2 \nu}$.

Then the scalar spectral index is $n_{s}-1=3-2 \nu$.

\section{B. Tensor perturbations}

Besides scalar perturbations, quantum fluctuations in gravitational waves form are produced as the same way. A linear perturbations in flat FLRW metric is given by

$$
d s^{2}=-d t^{2}+\left(\delta_{i j}+h_{i j}\right) a^{2}(t) d x^{i} d x^{j},
$$

the perturbation quantity $h_{i j}$ is gauge invariant. Since the energy-momentum tensor is diagonal, it has no contribution to the tensor perturbation equations; in other words, the tensor mode equation has no source term. The action for the corresponding equation is given by $[33,54]$

$$
S=\frac{1}{2 G} \int d^{4} x \sqrt{-g} \frac{1}{2} \partial_{\mu} h_{i j} \partial^{\mu} h^{i j},
$$

which has the same form as the action of the massless scalar field. Utilizing $u_{k}=a h_{k} / \sqrt{2} G$, and repeating the same process, the following equation will be concluded,

$$
\frac{d^{2} u_{k}(\tau)}{d \tau^{2}}+\left(k^{2}-\frac{1}{a} \frac{d^{2} a}{d \tau^{2}}\right) u_{k}(\tau)=0,
$$

for which the solution is now familiar. On the superhorizon limit, it reads as

$$
\left|u_{k}\right|=\left(\frac{H}{2 \pi}\right)\left(\frac{k}{a H}\right)^{-\nu_{T}+3 / 2}, \quad \nu_{T}=\frac{3}{2}-\epsilon .
$$

In turn, the tensor power spectrum at this limit is obtained as

$$
\mathcal{P}_{T}=64 \pi G\left(\frac{H}{2 \pi}\right)^{2}\left(\frac{k}{a H}\right)^{n_{T}},
$$

and the tensor spectral index is $n_{T}=3-2 \nu_{T}$. The tensor perturbations are detected indirectly through measuring the tensor-to-scalar ratio parameter $r=\mathcal{P}_{T} / \mathcal{P}_{s}$. Although no exact value for the parameter has been measured, there is only an upper bound so far.

\section{CONSISTENCY WITH OBSERVATION}

The model was studied generally; however, to get the best solution and in order to confirm the validity of the model, its prediction should be compare with observational data. The scalar spectral index in terms of $\eta$ is read by

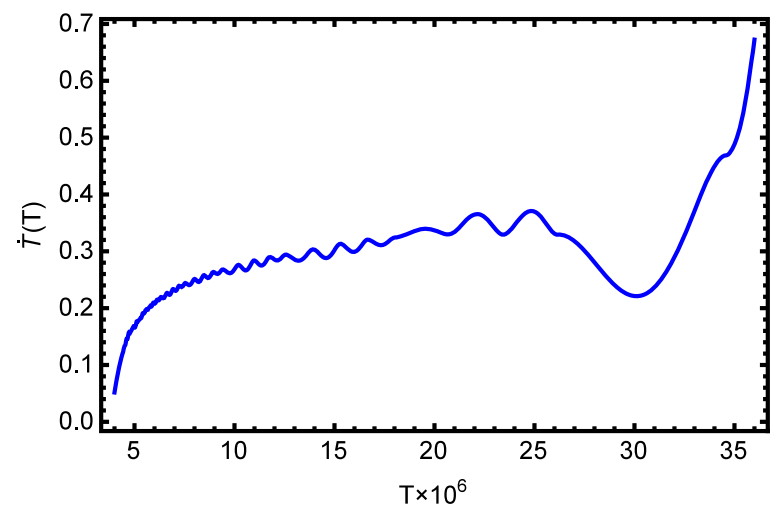

FIG. 4. The time derivative of the tachyon field versus the field is plotted during the inflation.

$$
\begin{aligned}
n_{s}-1 & =3-2 \nu=-2 \eta, \\
\nu^{2} & =\frac{9}{4}+3 \epsilon+3 \eta+\eta^{2}-\frac{2}{3} \epsilon \eta+2 \eta^{2} \epsilon
\end{aligned}
$$

in which $\eta=\beta$. According to the Planck observational data, the scalar spectral index is about $n_{s}=0.9666 \pm$ $0.003368 \% \mathrm{CL}$ [55]. Equation (30) indicates that there are two values for $\beta$ as $\beta \approx 0.0153$ and $\beta \approx-2.989$ to achieve the proper value for $n_{s}$. The latter choice for $\beta$ leads to a negative Hubble parameter which is not acceptable. For $\beta \approx 0.0153$, the time derivative of tachyon field is positive. Figure 4 illustrates the behavior of $\dot{T}$ versus the tachyon field, showing $\dot{T}>0$ during the inflation and denoting that the tachyon field at the end of inflation is bigger than at the horizon crossing. The slow-roll parameter $\epsilon$ is depicted in Fig. 5, where it is clear that, for small values of the field, $\epsilon$ is smaller than unity corresponding to the horizon crossing time. It first grows slowly for the smaller field, and then the growth becomes faster for bigger values of the tachyon field. Finally, it reaches one, indicating the end of inflation.

Using Eq. (30), the amplitude of scalar and tensor perturbations (25) and (29), one could find the behavior

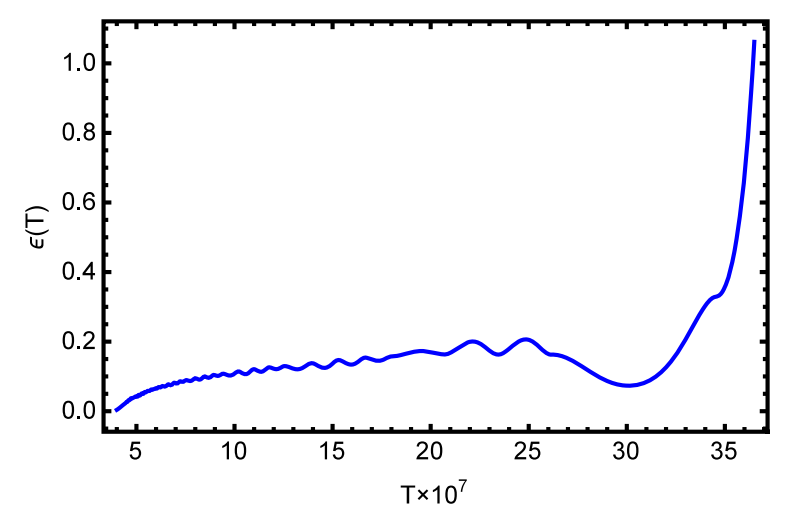

FIG. 5. The slow-roll parameters $\epsilon$ versus tachyon field during inflation. 


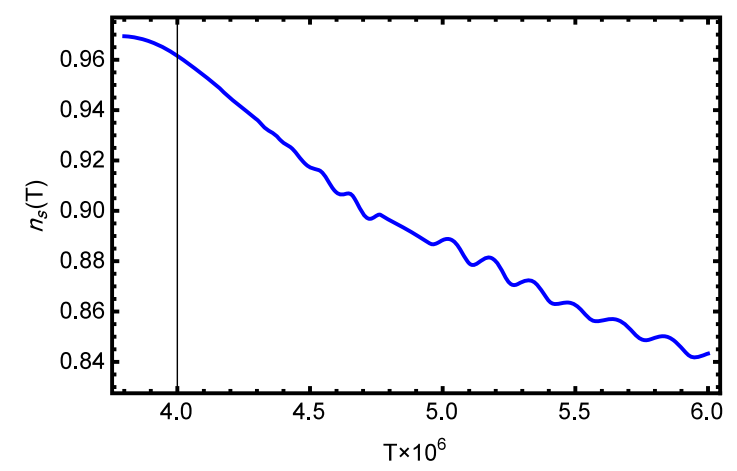

(a)

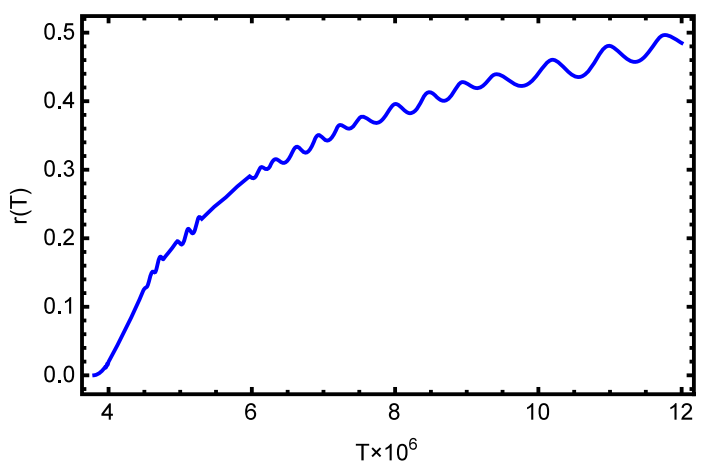

(b)

FIG. 6. The behavior of (a) scalar spectral index and (b) tensor-to-scalar ratio versus the tachyon field during the inflationary times.

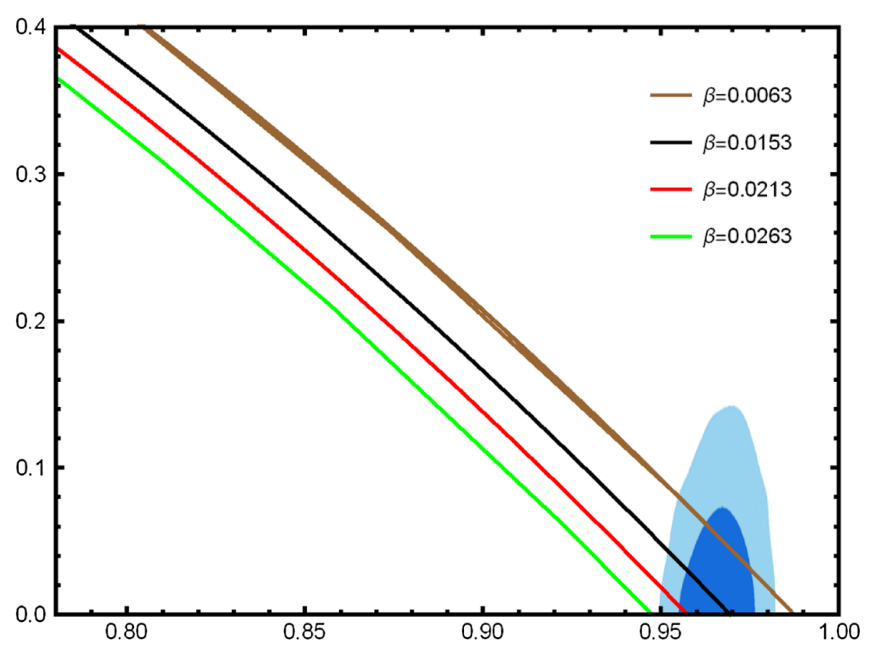

FIG. 7. The $r-n_{s}$ diagram.

of the scalar spectral index and tensor-to-scalar ratio $r$ during the inflation times. A proper model of inflation should have an acceptable consistency with observational data. According to the Planck data, there are $n_{s}=$ $0.9645 \pm 0.0049$ for scalar spectral index, and only an upper bound for the tensor-to-scalar ratio as $r<0.11$ [55].

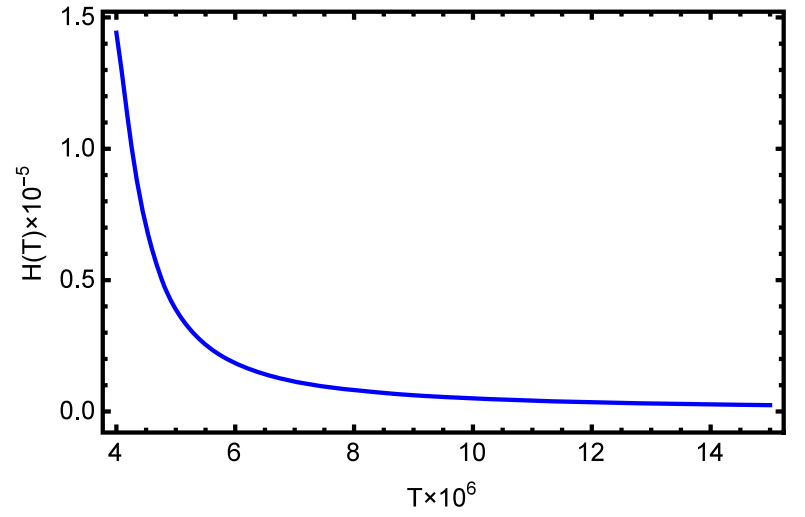

(a)
The behavior of these two perturbation parameters in our model is presented in Fig. 6. The scalar spectral index versus the tachyon field is depicted in Fig. 6(a), where it is clearly seen that, for smaller values of the tachyon field, this parameter could stand in an estimated range, and that with passing time and an increasing tachyon field, $n_{s}$ decreases as well. Figure 6(b) describes the behavior of the tensor-toscalar ratio versus the tachyon field, and it determines that, for smaller values of the tachyon field, the parameter $r$ could stand in an acceptable range. In contrast to the behavior of $n_{s}$, by passing time and the enhancement of the tachyon field, tensor-to-scalar ratio increases. To have a better comparison with observational data, Fig. 7 illustrates the $r-n_{s}$ diagram for four different values of the parameter $\beta$. The plot has been placed on the corresponding diagram of the Planck paper so that the blue era is the $68 \% \mathrm{CL}$ region and the light blue era is $95 \% \mathrm{CL}$ region. The figure shows that, for the smaller values of the tachyon field, the curves approach our interested regions in which $\beta \approx 0.0153$, which properly stands in the $68 \% \mathrm{CL}$ region. According to this figure, if we take $\beta=0.0153$ as the best choice, smaller values of $\beta$ diverts the curve to the right-hand side of planck region and bigger values of $\beta$ guides the line to the left-hand side. By passing times and

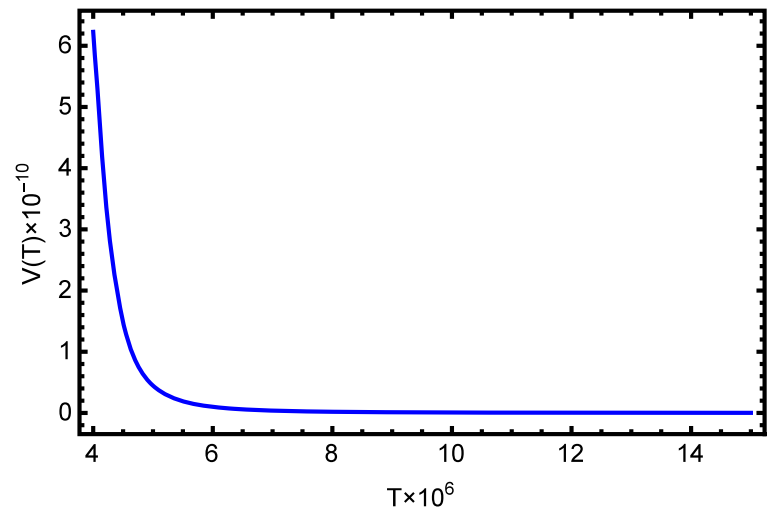

(b)

FIG. 8. (a) The Hubble parameter and (b) the potential versus tachyon field during inflation. 
enhancement of tachyon field, $n_{s}$ decreases and $r$ increases, and they recede from the Planck region. In order to have a numerical inside about the model predictions, it could be obtained that for $\beta \approx 0.0153$ there are the scalar spectral index $n_{s} \approx 0.9615$, the amplitude of scalar perturbations $\mathcal{P}_{s} \approx 2.13 \times 10^{-9}$, and the tensor-to-scalar ratio $r \approx 0.0197$, however these results are obtained for about $N=121 e$-folds.

The Hubble parameters and also the potential of tachyon field is shown in Fig. 8, where it could be realized that the energy scale of inflation at horizon crossing is about $V^{1 / 4} \approx 10^{-2.5}$. Inflation begins for smaller values of the tachyon field, and by passing time, the field becomes larger and rolls down from the top of the potential.

\section{VERY SMOOTH-ROLL TACHYON INFLATION}

In the canonical scalar field model of constantroll inflation, for $\beta=-3$ there is a flat potential i.e. $V^{\prime}(\phi)=0$ [24,25]. However, in the tachyon inflation, this choice does not lead to a flat potential; as a matter of fact, for $\beta=-3$, we have

$$
\frac{V^{\prime}(T)}{V(T)}=\frac{3 H \dot{T}^{3}}{1-\dot{T}^{2}}
$$

and this is due to the different form of the equation of motion of the tachyon field. On the other hand, for $\eta=$ $\beta\left(1-\dot{T}^{2}\right)$ and $\beta=-3$, a flat potential is concluded. This choice for $\eta$ is not constant; actually, it varies slowly, and for this reason we call this choice the "very smooth-roll," because it could be written in terms of the slow-roll parameter $\epsilon$ as $\eta=\beta(1-2 \epsilon / 3)$.

In this case, the differential equation for the Hubble parameters changes a little and is

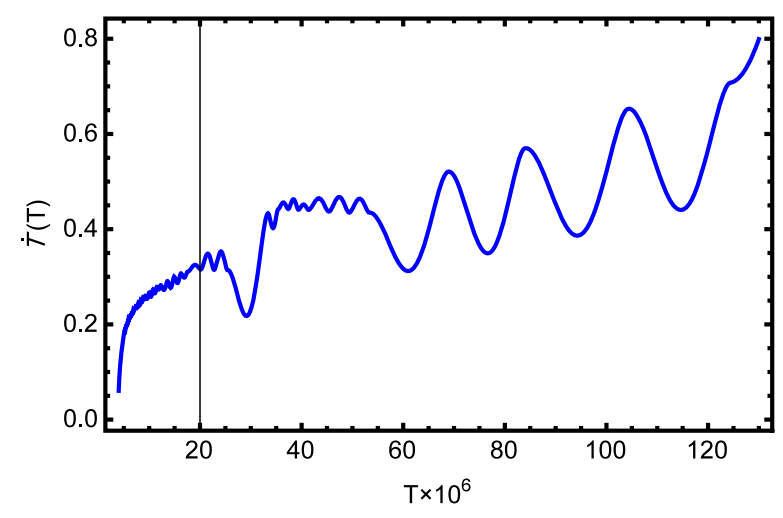

(a)
$H(T) \frac{d^{2} H(T)}{d T^{2}}-2\left(1+\frac{\beta}{3}\right)\left(\frac{d H(T)}{d T}\right)^{2}+\frac{3}{2} \beta H^{4}(T)=0$

however, all of the results we have obtained in Sec. III will be right even for this choice of $\eta$; the only difference is in the parameter $\nu$ as

$$
\nu^{2}=\frac{9}{4}+9 \epsilon-3 \beta-4 \beta \epsilon+\beta^{2}+2 \beta^{2} \epsilon,
$$

which in turns appears in the scalar spectral index so that up to the first order of $\epsilon$, there is

$$
n_{s}-1=3-2 \nu \text {. }
$$

It seems that for any value of $n_{s}$, there are two values for $\beta$. For $\beta \approx 0.014$, the time derivative of the tachyon field is positive, as illustrated in Fig. 9(a). The slow-roll parameter $\epsilon$ is smaller than unity at the horizon crossing time. By passing time, the tachyon field increases and $\epsilon$ also approaches one. Its behavior is shown in Fig. 9(b), where an almost oscillating behavior for the $\epsilon$ is clear; however, it grows up generally by passing time and increasing the tachyon field. The parameter finally reaches one and inflation ends. From the definition of the $\eta$, this parameter has a decreasing behavior by increasing $\epsilon$ which has been illustrated in Fig. 10.

The amplitude of scalar and tensor perturbation can be read from Eqs. (25) and (29), and, by applying the scalar spectral index for this case from Eq. (32), one can figure out the behavior of the main perturbation parameters $n_{s}$ and $r$ during the inflationary times. Figure 11 exhibits the scalar spectral index versus the tachyon field during the inflation. The general behavior is the same as the previous case so that $n_{s}$ decreases by approaching the end of inflation. It stands in the acceptable range proposed by Planck for

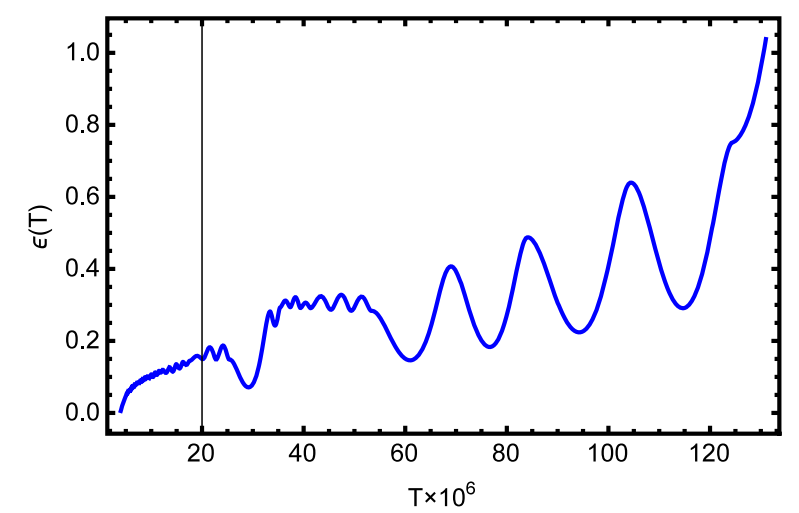

(b)

FIG. 9. (a) The time derivative of tachyon field, and (b) the behavior of the slow-roll parameter $\epsilon$ are plotted versus the field during the inflation. 


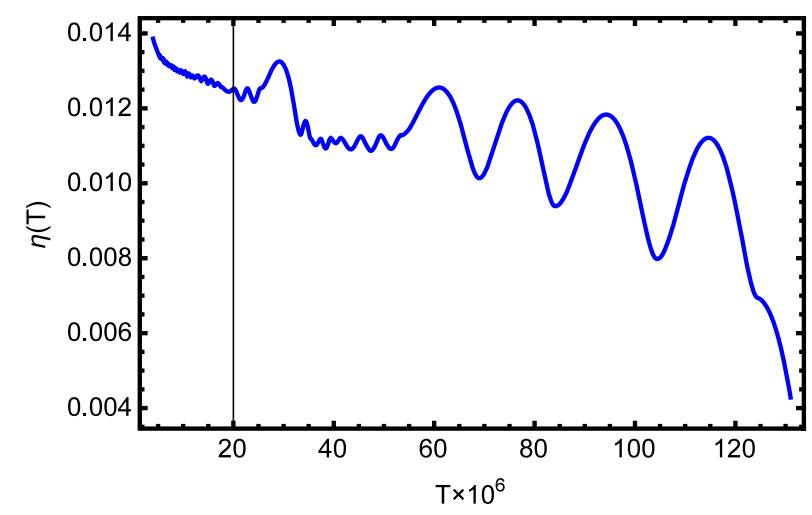

FIG. 10. The behavior of the parameter $\eta$ are plotted versus the field during the inflation.

smaller values of the field. The general behavior of $r$ is different in that as it approaches the end of inflation, it increases, as illustrated in Fig. 11(b). The parameter $r$ could be used for smaller values of the field, which is consistent with the results obtained for $n_{s}$. In order to emphasize this consistency, the $r-n_{s}$ diagram for the model is plotted in Fig. 12 for different values of $\beta$, where this diagram is placed on the corresponding diagram of Planck to clarify this agreement between the model prediction and observational data. If one takes $\beta=0.014$ as the best choice, the smaller and bigger values divert the lines, respectively, to the right- and left-hand side of the Planck region. In order to have numerical insight for this case, for $\beta \approx 0.014$, the scalar spectral index is about $n_{s} \approx 0.9615$, the amplitude of scalar perturbations is about $\mathcal{P}_{s} \approx 2.13 \times 10^{-9}$, and the tensor-to-scalar ratio is predicted about $r \approx 0.0272$, for about $N=126 e$-folds.

The Hubble parameter is displayed in Fig. 13(a), and Fig. 13(b) shows the potential of tachyon field. From the last figure, one can find out that the energy scale of inflation at the time of horizon crossing is $V^{1 / 4} \approx 1.7 \times 10^{-2.5}$ (about $0,005 \mathrm{M}_{\mathrm{p}}$ ) which is on the same order as the energy scale of the previous case.

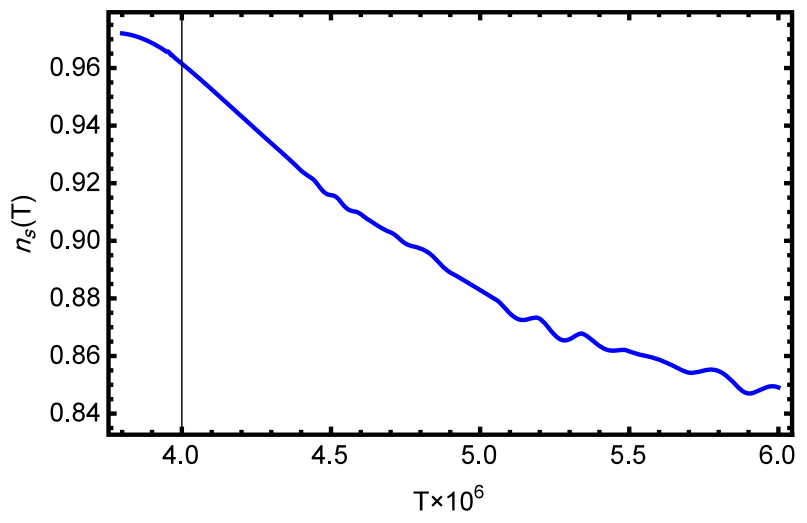

(a)

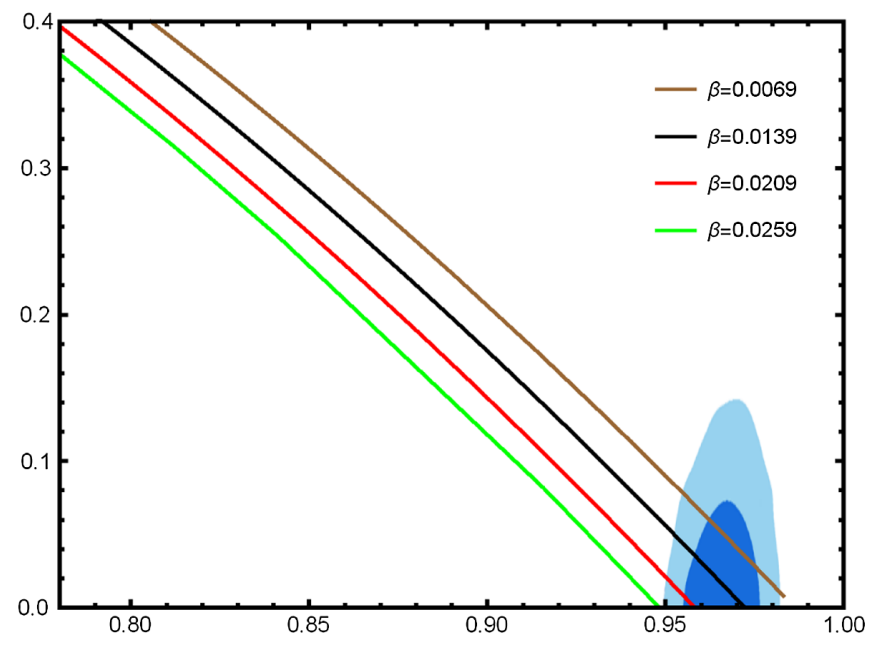

FIG. 12. The $r-n_{s}$ diagram of the model.

\section{ATTRACTOR BEHAVIOR}

Although the exact analytical solution for the model was not derived, the numerical solution for the Hubble parameter enabled us to consider the various features of the model and its consistency with observational data. In this section, the attractor behavior of the solution will be investigated. Since a solution to the differential equation might not be attractor, this feature explicitly will be examined. In this regard, the same strategy of $[42,43,56]$ is used, and the homogenous perturbation $\delta H(T)$ is taken to the solution $H_{0}(T)$. Equation (8) gives the scalar potential in terms of the Hubble parameters and its first derivative. Inserting $H(T)=H_{0}(T)+\delta H(T)$ in Eq. (8), and keeping only the first order of the perturbation, one arrives at

$$
\frac{\epsilon(T)}{3-2 \epsilon(T)} \frac{\delta H^{\prime}(T)}{H^{\prime}(T)}=\frac{3}{3-2 \epsilon(T)} \frac{\delta H(T)}{H(T)} .
$$

Integrating from the above expression, the perturbation parameter $\delta H(T)$ during inflation is obtained as

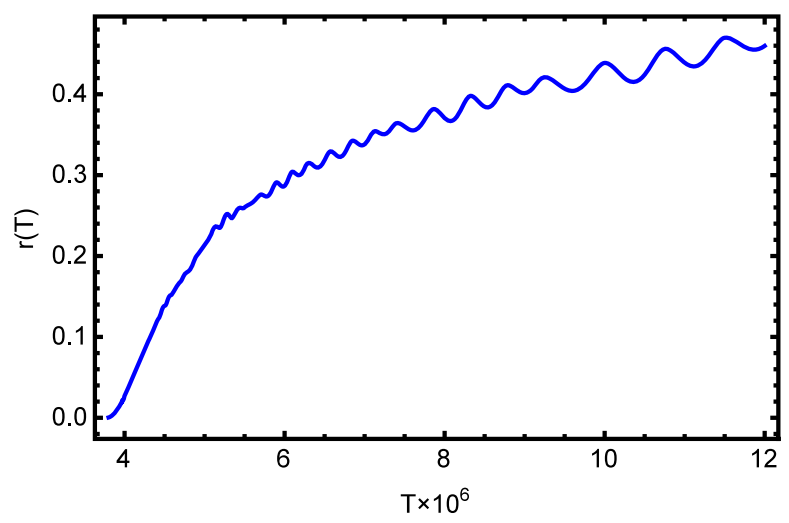

(b)

FIG. 11. (a) The scalar spectral index, and (b) the tensor-to-scalar ratio versus the tachyon field during the inflationary times. 


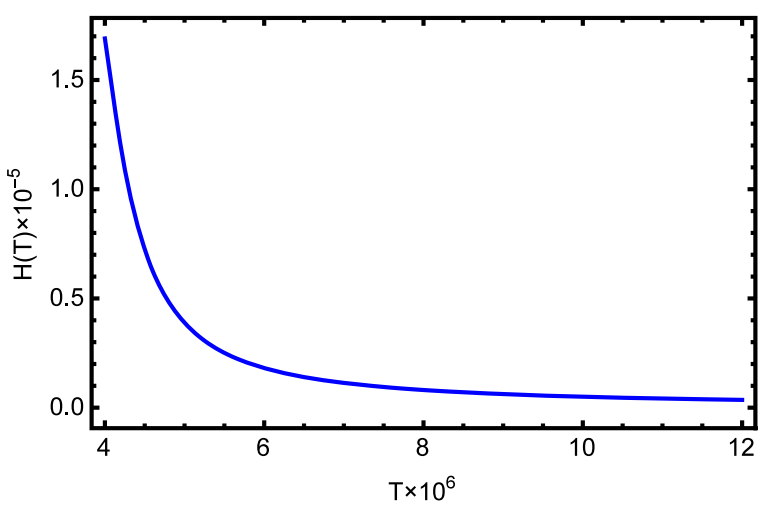

(a)

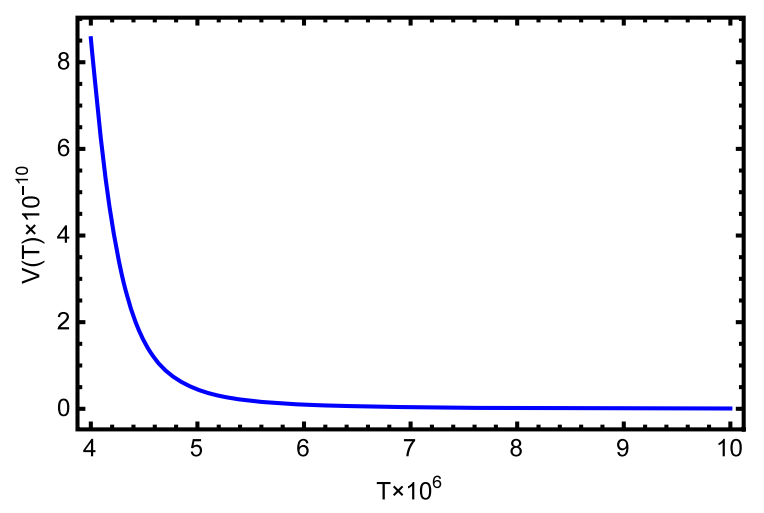

(b)

FIG. 13. (a) The Hubble parameter, and (b) the potential of scalar field are plotted versus the field during the inflation.

$$
\begin{aligned}
\delta H(T) & =\delta H\left(T_{i}\right) \exp \left[\int_{T_{i}}^{T} \frac{3}{\epsilon(T)} \frac{H^{\prime}(T)}{H(T)} d T\right] \\
& =\delta H\left(T_{i}\right) \exp \left[\int_{T_{i}}^{T} \frac{9}{2} \frac{H^{3}(T)}{H^{\prime}(T)} d T\right],
\end{aligned}
$$

in which $\delta H\left(T_{i}\right)$ is the perturbation parameter at the initial time. It is clear that the power of the exponential term is related to the number of $e$-fold expressions, so that

$$
\delta H(T)=\delta H_{0}(T) \exp [-3 N(T)]
$$

To understand the behavior more precisely, the integrand in the power of the exponential term is depicted in Fig. 14, in which the colored area is the integral term. The plots display that by approaching to the end of inflation the colored area becomes larger but with negative sign. In other words, by passing time the perturbation parameter becomes smaller and smaller. Therefore, the solution of the model possesses the attractor behavior.

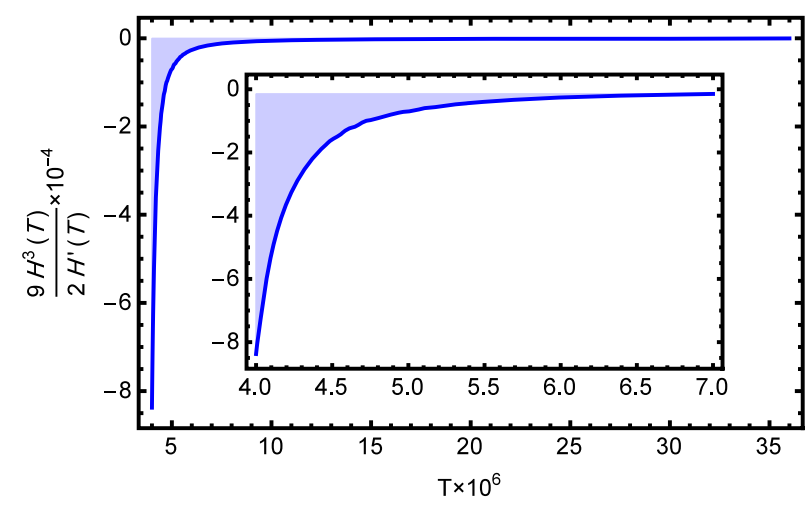

(a)

\section{END OF INFLATION}

Inflation is known as an accelerated expansion phase; namely, the Universe has a positive acceleration and it grows extremely in short period of time. The acceleration equation for the Universe is given by

$$
\frac{\ddot{a}}{a}=-(\rho+3 p)=-(1+3 \omega) \rho
$$

in which $\omega=p / \rho$. Using Eq. (5), there is

$$
\frac{\ddot{a}}{a}=H^{2}\left(1-\frac{3}{2} \dot{\phi}^{2}\right)
$$

To have inflation, the equation of state parameter must be bigger than $\frac{-1}{3}$ or, in other words, $\dot{\phi}^{2}<\frac{2}{3}$. Figure 15(a) displays the behavior of the equation of state parameter $\omega$ during the inflationary times. At the horizon exit time, there is $\omega \simeq-1$, then we have a quasi-de Sitter expansion. By growing the tachyon field, $\omega$ becomes bigger continuously and crosses the points $\omega=-1 / 3$ and $\omega=0$ that indicates that the Universe enters a deceleration phase known as a

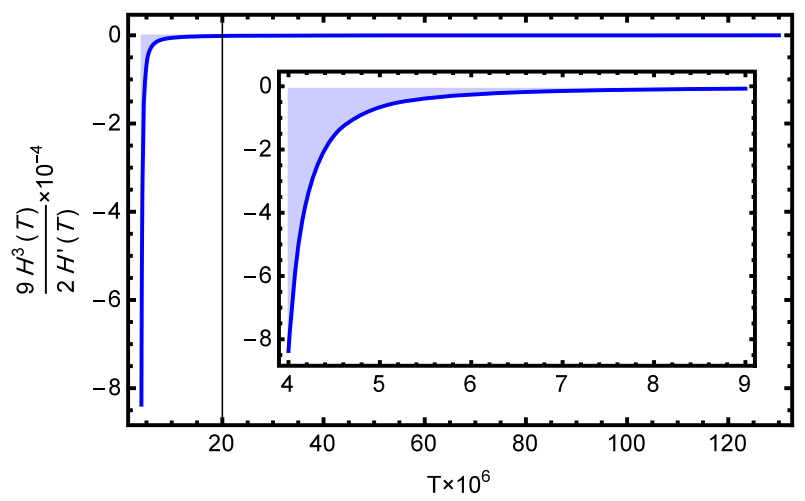

(b)

FIG. 14. Behavior of the integrand, $\frac{9}{2} \frac{H^{3}(T)}{H^{\prime}(T)}$, in terms of tachyon field during inflation for (a) first case related to Sec. IV; (b) second case related to Sec. V. 


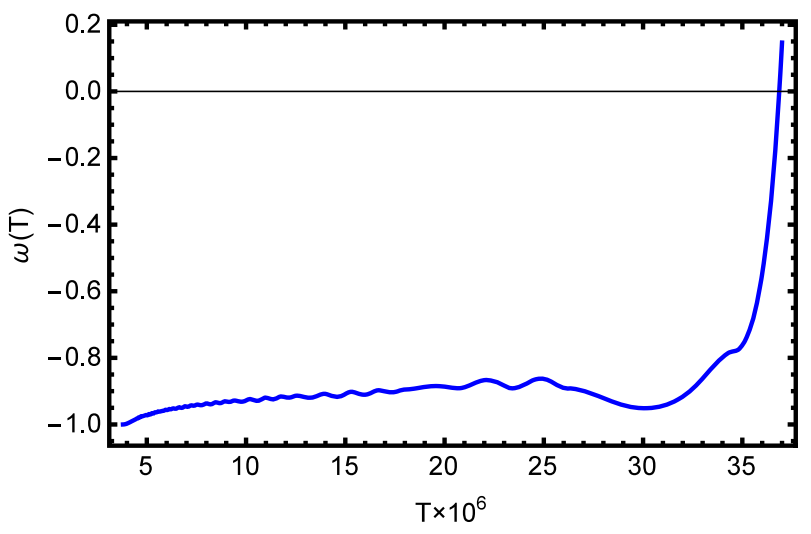

(a)

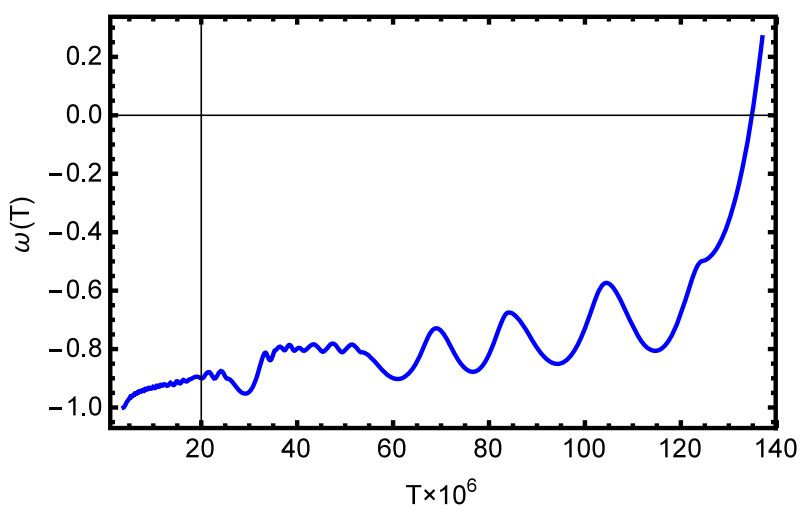

(c)

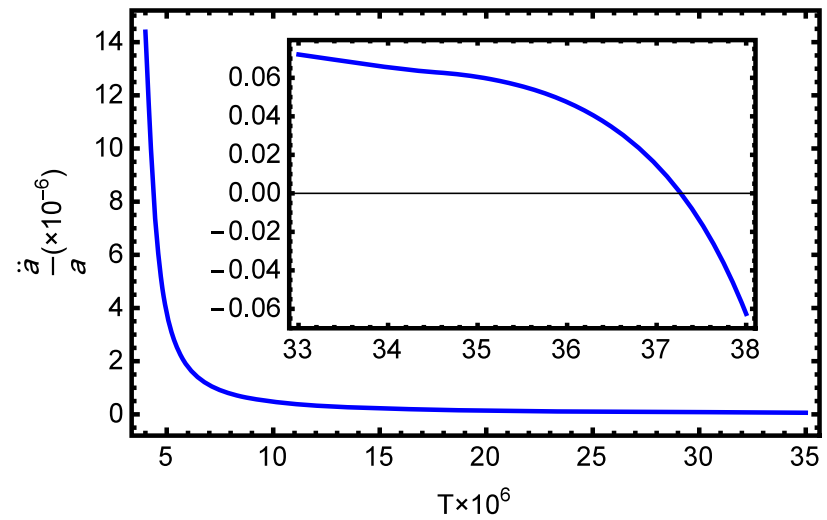

(b)

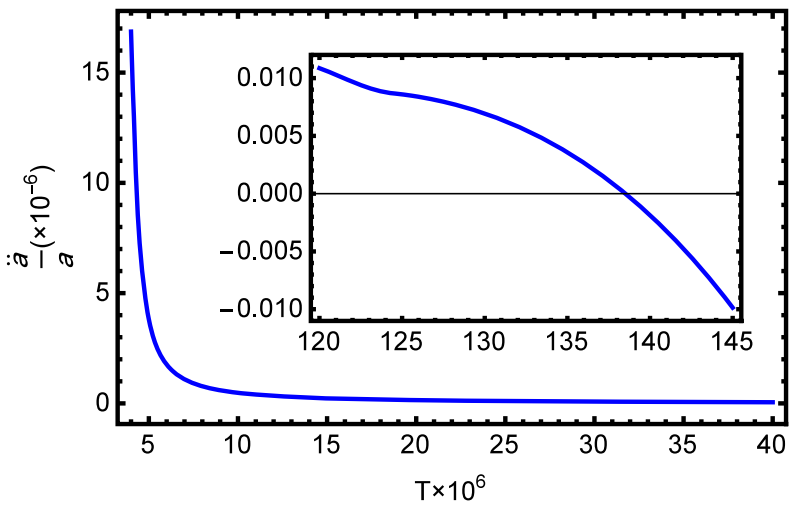

(d)

FIG. 15. For the first case: (a) The equation of state parameter and (b) acceleration of the Universe versus scalar field. Figures (c) and (d) show, respectively, the same parameters for the second case.

radiation phase. Figure 15(b) is another expression for this fact that the Universe transits from an acceleration phase $(\ddot{a}>0)$ to a deceleration phase $(\ddot{a}<0)$. Therefore, the model could successfully describe a graceful exit from the inflationary phase.

The same process happens for the second case as determined in Figs. 15(c) and 15(d). The equation of state parameter is near -1 at the beginning and it grows by increasing of the tachyon field and crosses the point $\omega=-1 / 3$ which indicates that the Universe undergoes a phase transition, namely from acceleration to deceleration phase that has been shown in Fig. 15(d).

\section{CONCLUSION}

Tachyon inflation was studied in constant-roll scenario where the second order of the slow-roll parameter is assumed as a constant. This choice leads to a nonlinear differential equation for the Hubble parameter, and it complicity form forced us to solve it numerically.

Since this constant taken for the second slow-roll parameter could be of order unity, one should reinvestigate the cosmological perturbations of the model. The cosmological perturbation was considered in the work, and for cosmological perturbation it was clear that there are some correction terms in the amplitude of scalar perturbations and also in the scalar spectral index. However, since the energymomentum tensor has no contribution in the tensor perturbations equations, and also because these equations only contain the first slow-roll parameter, there was no change in the amplitude of tensor perturbation with respect to the slowroll inflationary model.

Obtaining the perturbation parameters and comparing them with observational data indicates that there is a specific choice for the constant parameter $\beta$ that makes the amplitude of scalar perturbation almost scale invariant in superhorizon scales. Taking $\beta \approx 0.024$, we come to a positive time derivative of the tachyon field which shows that the field grows during the inflationary times, which is consistent with Fig. 5 where the behavior of the slow-roll parameters $\epsilon$ is plotted and clearly shows that inflation ends for bigger values of the field. The amplitude of scalar perturbations, scalar spectral index, and tensor-to-scalar ratio for the selected $\beta$ are, respectively, obtained as $0.9615,2.13 \times 10^{-9}$, and 0.0197 for about $121 e$-folds. 
As another case, the second slow-roll parameter was taken as $\eta=\beta\left(1-\dot{T}^{2}\right)$ so that for $\beta=-3$, there is flat potential for the model in analogues with the canonical scalar field model that we have a flat potential for $\eta=-3$. In this case, the parameter $\eta$ is not exactly constant, and it varies slowly. The differential equation for the Hubble parameter changes a little with respect to the previous case. Calculating the perturbation equations shows that there are only some correction terms in the parameter $\nu$, which in turn comes to some correction terms in the equation of the scalar spectral index. By taking $\beta \approx 0.0233$, the time derivative of the field is positive, indicating an increasing behavior for the magnitude of the field, which displays that there is an end for inflation for the bigger values of the field than the horizon crossing time, as illustrated in Fig. 9(b). The results state that $n_{s} \approx 0.9645, \mathcal{P}_{s} \approx 2.19 \times 10^{-9}$, and $r \approx 0.0272$ with about $126 e$-folds.
The next section was related to studying the attractor behavior of the solution of the model. The results determined that the perturbation parameter $\delta H(T)$ decreases exponentially toward the end of inflation, which indicates that the solution of the Hubble parameter has attractor behavior. Finally, the subject of the exit from inflation was considered. In this regard, the equation of state parameter and acceleration of the Universe were depicted versus the scalar field. It was specified that the equation of state parameter is near -1 at the beginning giving a quasi-de Sitter expansion. By enhancement of the tachyon field the parameter grows continuously and crosses the point $\omega=-1 / 3$ toward the bigger values indicating a negative acceleration for the Universe. This fact was confirmed by the acceleration plot, too, where we realized that the acceleration parameter moves from positive to negative, indicating that the Universe exits from the acceleration phase (inflation) to a deceleration phase.
[1] A. H. Guth, Phys. Rev. D 23, 347 (1981).

[2] A. D. Linde, Phys. Lett. B 108, 389 (1982).

[3] A. Albrecht and P. J. Steinhardt, Phys. Rev. Lett. 48, 1220 (1982).

[4] A. D. Linde, Phys. Lett. B 129, 177 (1983).

[5] C. Armendariz-Picon, T. Damour, and V.F. Mukhanov, Phys. Lett. B 458, 209 (1999).

[6] J. Garriga and V.F. Mukhanov, Phys. Lett. B 458, 219 (1999).

[7] R. Maartens, D. Wands, B. A. Bassett, and I. P. C. Heard, Phys. Rev. D 62, 041301 (2000).

[8] T. Golanbari, A. Mohammadi, and Kh. Saaidi, Phys. Rev. D 89, 103529 (2014).

[9] K.-i. Maeda and K. Yamamoto, J. Cosmol. Astropart. Phys. 12 (2013) 018.

[10] A. A. Abolhasani, R. Emami, and H. Firouzjahi, J. Cosmol. Astropart. Phys. 05 (2014) 016.

[11] S. Alexander, D. Jyoti, A. Kosowsky, and A. Marciano, J. Cosmol. Astropart. Phys. 05 (2015) 005.

[12] M. Tirandari and Kh. Saaidi, Nucl. Phys. B925, 403 (2017).

[13] A. Berera, Phys. Rev. Lett. 75, 3218 (1995).

[14] A. Berera, Nucl. Phys. 585, 666 (2000).

[15] A. N. Taylor and A. Berera, Phys. Rev. D 62, 083517 (2000).

[16] L. M. H. Hall, I. G. Moss, and A. Berera, Phys. Rev. D 69 , 083525 (2004).

[17] M. Bastero-Gil and A. Berera, Phys. Rev. D 71, 063515 (2005).

[18] K. Sayar, A. Mohammadi, L. Akhtari, and Kh. Saaidi, Phys. Rev. D 95, 023501 (2017).

[19] L. Akhtari, A. Mohammadi, K. Sayar, and Kh. Saaidi, Astropart. Phys. 90, 28 (2017).

[20] A. D. Linde, Particle Physics and Inationary Cosmology (Harward Academic, Chur, Switzerland, 1990).
[21] S. Weinberg, Cosmology (Oxford University Press, New York, 2008).

[22] W. H. Kinney, Phys. Rev. D 72, 023515 (2005).

[23] M. H. Namjoo, H. Firouzjahi, and M. Sasaki, Europhys. Lett. 101, 39001 (2013).

[24] J. Martin, H. Motohashi, and T. Suyama, Phys. Rev. D 87, 023514 (2013).

[25] H. Motohashi, A. A. Starobinsky, and J. Yokoyama, J. Cosmol. Astropart. Phys. 09 (2015) 018.

[26] W. H. Kinney, Phys. Rev. D 56, 2002 (1997).

[27] D. S. Salopek and J. M. Stewart, Classical Quantum Gravity 9, 1943 (1992).

[28] A. R. Liddle, P. Parsons, and J. D. Barrow, Phys. Rev. D 50, 7222 (1994).

[29] Z.-K. Guo, Y.-S. Piao, R.-G. Cai, and Y.-Z. Zhang, Phys. Rev. D 68, 043508 (2003).

[30] A. Aghamohammadi, A. Mohammadi, T. Golanbari, and Kh. Saaidi, Phys. Rev. D 90, 084028 (2014).

[31] Kh. Saaidi, A. Mohammadi, and T. Golanbari, Adv. High Energy Phys. 2015, 1 (2015).

[32] H. Sheikhahmadi, E. N. Saridakis, A. Aghamohammadi, and K. Saaidi, J. Cosmol. Astropart. Phys. 10 (2016) 021.

[33] D. Baumann, arXiv:0907.5424v2.

[34] S. Nojiri, S. D. Odintsov, and V. K. Oikonomou, Classical Quantum Gravity 34, 245012 (2017).

[35] H. Motohashi and A. A. Starobinsky, Eur. Phys. J. C 77, 538 (2017).

[36] A. Awad, W. E. Hanafy, G. G. L. Nashed, S. D. Odintsov, and V. K. Oikonomou, arXiv:1710.00682.

[37] L. Anguelova, P. Suranyi, and L. C. R. Wijewardhana, J. Cosmol. Astropart. Phys. 02 (2018) 004.

[38] Q. Gao, Y. Gong, and Q. Fei, arXiv:1801.09208.

[39] S. D. Odintsov, V. K. Oikonomou, and L. Sebastiani, Nucl. Phys. B923, 608 (2017).

[40] V. K. Oikonomou, Mod. Phys. Lett. A 32, 1750172 (2017). 
[41] A. Karam, L. Marzola, T. Pappas, A. Racioppi, and K. Tamvakis, arXiv:1711.09861v1.

[42] S. D. Odintsov and V. K. Oikonomou, J. Cosmol. Astropart. Phys. 04 (2017) 041.

[43] S. D. Odintsov and V. K. Oikonomou, Phys. Rev. D 96, 024029 (2017).

[44] V. K. Oikonomou, arXiv:1709.02986.

[45] A. Sen, J. High Energy Phys. 04 (2002) 048.

[46] A. Sen, J. High Energy Phys. 07 (2002) 065.

[47] A. Sen, Mod. Phys. Lett. A 17, 1797 (2002).

[48] G. W. Gibbons, Phys. Lett. B 537, 1 (2002).

[49] M. Fairbairn and M. H. G. Tytgat, Phys. Lett. B 546, 1 (2002).
[50] S. Mukohyama, Phys. Rev. D 66, 024009 (2002).

[51] A. Feinstein, Phys. Rev. D 66, 063511 (2002).

[52] T. Padmanabhan, Phys. Rev. D 66, 021301 (2002).

[53] V. F. Mukhanov, H. A. Feldman, and R. H. Brandenberger, Phys. Rep. 215, 203 (1992).

[54] A. Riotto, ICTP Lect. Notes Ser. 14, 317 (2003).

[55] P. A. R. Ade et al. (Planck Collaboration), Astron. Astrophys. 594, A20 (2016).

[56] D. H. Lyth and A. R. Liddle, The Primordial Density Perturbation (Cambridge University Press, Cambridge, England, 2009). 